\title{
Growth of Germanium Nanowires on a Flexible Organic Substrate
}

\author{
Lauren A. Klein ${ }^{1}$, Daniel D. T. Mastrogiovanni ${ }^{1}$, Aurelien Du Pasquier ${ }^{2}$, \\ Tong Wang, Alan S. Wan ${ }^{1}$ and Eric Garfunkel ${ }^{1}$ \\ ${ }^{1}$ Department of Chemistry and Chemical Biology, Rutgers University, Piscataway, \\ ${ }^{2}$ Department of Materials Science and Engineering, Rutgers University, Piscataway, \\ New Jersey, \\ USA
}

\section{Introduction}

Germanium nanowires (GeNWs) have received extensive attention due to their potential use in electronic, optoelectronic, sensor and other applications. (Heath 2000; Wu and Yang 2000; Hanrath and Korgel 2002; Hanrath and Korgel 2004) (Greytak 2004) The small size, high aspect ratio, and high surface/volume ratio of Ge and other semiconducting nanowires also make them of great fundamental scientific interest. (Jing, Ni et al. 2006; Allen 2007) GeNWs grown via a vapor-liquid-solid (VLS) mechanism in a modified chemical vapor deposition (CVD) process are crystalline. (Dailey 2004) They can be patterned, functionalized, and/or made components of heterostructures for device applications. (Yun 2003; Hanrath and Korgel 2004; Dai 2005; Wang, Chang et al. 2005; Adhikari, Marshall et al. 2006; Pasquier, Mastrogiovanni et al. 2007) Germanium possesses a higher carrier mobility than silicon, as well as a lower nanowire growth temperature when grown using the VLS method (Adhikari, Marshall et al. 2007).

There would be a large market for flexible electronic and photonic devices if functionality and performance could approach that of conventional solid state devices at a reasonable cost. (Bradley, Gabriel et al. 2003; Bitetti, Marchetti et al. 2007) Polymer films hold great promise as a cost-efficient substrate for potential applications such as those of photovoltaics or display technologies, due in large part to their ease of manufacturing, low cost, and extensive past development.

Other complex materials combinations are being explored including integrating inorganic nanoparticles or small molecule organics with polymers. Two classes of methods for integrating semiconductor nanowires with flexible materials are: (i) transferring wires from a sacrificial growth substrate to a new material via contact printing or solution deposition (Bradley, Gabriel et al. 2003; McAlpine, Friedman et al. 2003; Saran, Parikh et al. 2004; Fan, Ho et al. 2008), and (ii) direct integration via solution growth or very low temperature VSS growth. (Gibson 2005; Lin 2005; Kang 2008) In the former method nanowires must first be grown on some other substrate and then harvested. However, for many large-scale applications direct growth would be highly preferable. Growing nanowires on the polymer substrates simplifies the processing of any devices through reduction of the number of 
necessary steps, and thus becomes more cost-effective. Direct growth saves time, reduces the risk of damage to the nanowires during harvesting, and also securely fastens the wires to the substrate. The wires grown in this study were anchored to the polymer with sufficient integrity to withstand extensive flexing of the substrate, and were manufactured at standard VLS growth temperatures.

The relatively low decomposition temperatures of most polymers cannot survive the growth temperature used in most VLS nanowire processing. However, the growth temperatures of GeNWs, $300-400^{\circ} \mathrm{C}$, does allow for direct growth on thermally stable polymers. Polyimides such as Kapton, are one class of polymers that offer promise. Recent advances in the thermal stability of polymers (to over $600^{\circ} \mathrm{C}$ ) offer hope that flexible, and in some cases transparent, substrates can be manufactured to enable the extension of our methods to other nanowire/nanotube growth systems such as silicon nanowires or carbon nanotubes. (Ree, Kim et al. 1997; Maya, Lozano et al. 2005; Li, Liu et al. 2006; Choi, Chung et al.; Chung and Hsiao 2008; Wang, Yi et al. 2008)

\section{Growth and processing methods}

\subsection{Polymer processing}

In the near future, it is expected that an increasing amount of these materials will be made commercially available, though not many are on the market today. For the studies contained herein, Kapton (Dupont) and poly[ $\left(4,4^{\prime}\right.$-hexafluoroisopropylidene) diphthalic anhydride-alt-3,6-diaminoacridine hemisulfate] (Sigma-Aldrich) are used. It is the relatively low growth temperature of germanium nanowires which makes growth on any polymer, even those listed above, possible. Both materials possess a glass-transition temperature just above the required growth range for germanium nanowires.

To prepare the fluorinated polyimide samples, poly[(4, $4^{\prime}$-hexafluoroisopropylidene $)$ diphthalic anhydride-alt-3,6-diaminoacridine hemisulfate] is dissolved in DMSO at a concentration of $10 \mathrm{mg} / \mathrm{mL}$. The solution is subsequently drop cast onto a glass slide while simultaneously heating at $50{ }^{\circ} \mathrm{C}$ to expedite solvent removal. It should be noted that by this preparation method, the fluorinated polyimide samples are not flexible, but if bulk material were available, flexible substrates of fluorinated polyimide could easily be treated in the same manner as Kapton.

Germanium nanowires grow via a catalyzed mechanism, and the polymer substrates must first be coated with gold a thin gold film or gold particles. Gold deposition is achieved by one of two methods, either through sputter coating thin films on the surface, or through wet chemical treatment. Kapton sheets were sputter-coated with Au films roughly 2, 5, 10, and 50 nm thick. Exact thicknesses were determined using Rutherford Backscattering Spectroscopy (RBS). Polymer substrates cleaned with methanol to remove organic contaminants were exposed to a $0.1 \mathrm{M}$ solution of 3-aminopropyltriethoxysilane (APTES) in methanol for 24 hours. The sheets were removed, rinsed clean, and then blown dry with purified $\mathrm{N}_{2}$. The functionalized films were then submerged in commercial gold nanoparticle solutions (Ted Pella, Inc) with nanoparticle diameters ranging between 10 and $50 \mathrm{~nm}$.

\subsection{Germanium nanowire growth}

The functionalized plastic substrates were confined to a specially designed stainless steel holder to prevent deformation of the polymer as higher temperatures were attained. For 
growth on Kapton a stainless steel holder was required to keep the thin plastic sheets from deforming during growth. The stainless steel holder we used to secure the Kapton is shown in figure 1, below. As our growth temperatures approach the glass transition temperature of Kapton, if the film was not restrained, significant curling of the film would take place, undesirable for subsequent processing and studies. The holder measures $10 \mathrm{~cm}$ $x 1.8 \mathrm{~cm} \times 0.6 \mathrm{~cm}$.

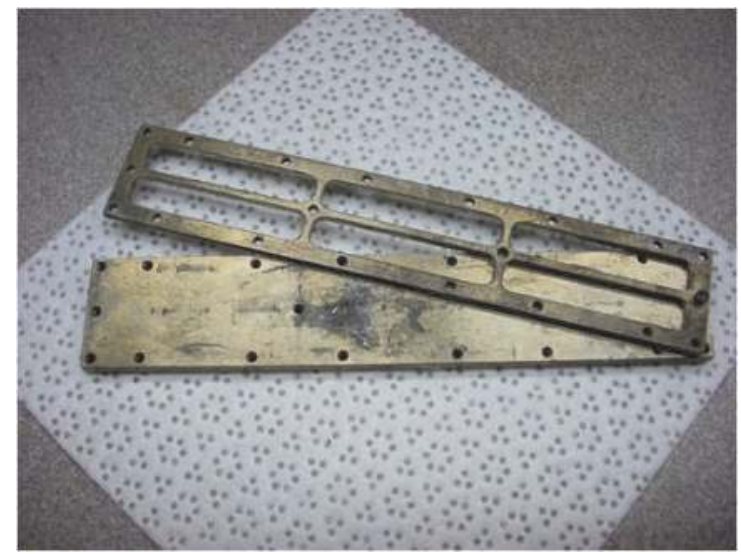

Fig. 1. Stainless steel holder designed to accommodate Kapton samples

All processed substrates were placed in a hot-wall CVD reactor. Germane $\left(\mathrm{GeH}_{4}\right)$ gas $(5 \%$ in He, Voltaix) was flowed through the system at a rate of $20 \mathrm{sccm}$ at partial pressures ranging from 1-3 Torr. Growth timing was varied between 5 and 30 minutes. Growth temperature was held at $360^{\circ}$. Proccessing temperatures in this range lead to conical wires, due to radial non-catalyzed CVD growth. A diagram of this procedure is shown in figure 2 .

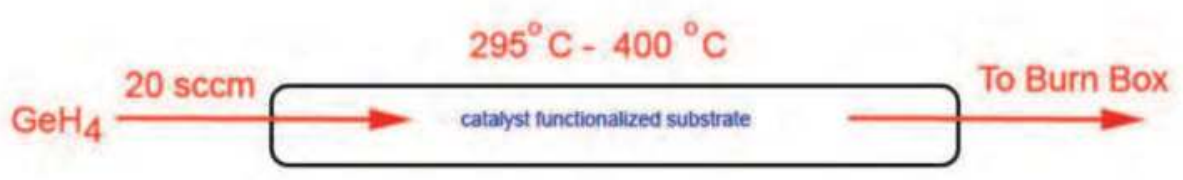

Fig. 2. Diagram of germanium nanowire growth scheme.

\subsection{Post growth processing}

Nanowire samples were placed in a $0.1 \mathrm{M}$ solution of 1-octanethiol for 24 hours immediately upon removal from the reactor. Alkanethiol solutions have been shown to form selfassembled monolayers on germanium substrates and are attractive chemicals to begin passivation studies with as they can be terminated with large number of functional groups which can be subsequently reacted to more fully functionalize a surface. (Hanrath and Korgel 2004; Wang, Chang et al. 2004; Wang, Chang et al. 2005) (Sang 2001) 


\section{Results}

\subsection{Growth on polymer substrates}

Germanium nanowires grown on Kapton and fluorinated polyimide samples produce a high yield of nanowires with good mechanical and chemical robustness. Wires produced in these growths are slightly tapered, due to uncatalyzed CVD deposition on the sides of the wire as growth proceeds.

A satisfactory mechanical robustness was observed when wires were anchored to a flexible material; the systems underwent considerable bending and flexing consistent with being handled in the lab and no detachment or damage to the wires was evident. Figure 3, below, represents one such system, imaged while the Kapton substrate was restricted in a bent position.

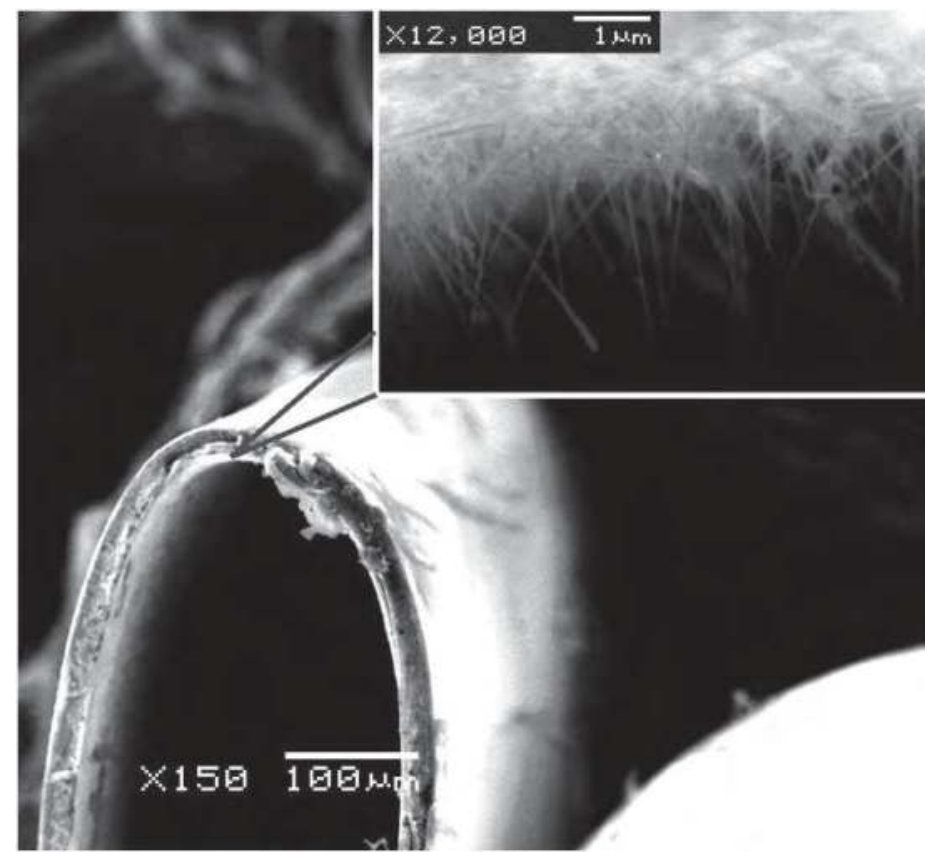

Fig. 3. Germanium nanowires grown on Kapton. Inset image shows wires from the height of the Kapton fold.

Figure 4 shows a side-view scanning electron micrograph of GeNWs on Kapton. These wires were grown at $360^{\circ}$ for 15 minutes from 10nm Au colloid particles. Remaining catalysts can be observed at the tips of the wires. Tapering is clearly evident, most likely stemming from non-catalyzed radial CVD growth.

In Figure 5, a top-view image is shown of GeNWs grown on poly[(4,4'hexafluoroisopropylidene) diphthalic anhydride-alt-3,6-diaminoacridine hemisulfate]. These wires were grown at $360^{\circ}$ for 15 minutes from $10 \mathrm{~nm}$ Au colloid particles. Excellent uniformity of the length and diameters of the wires is noted. 


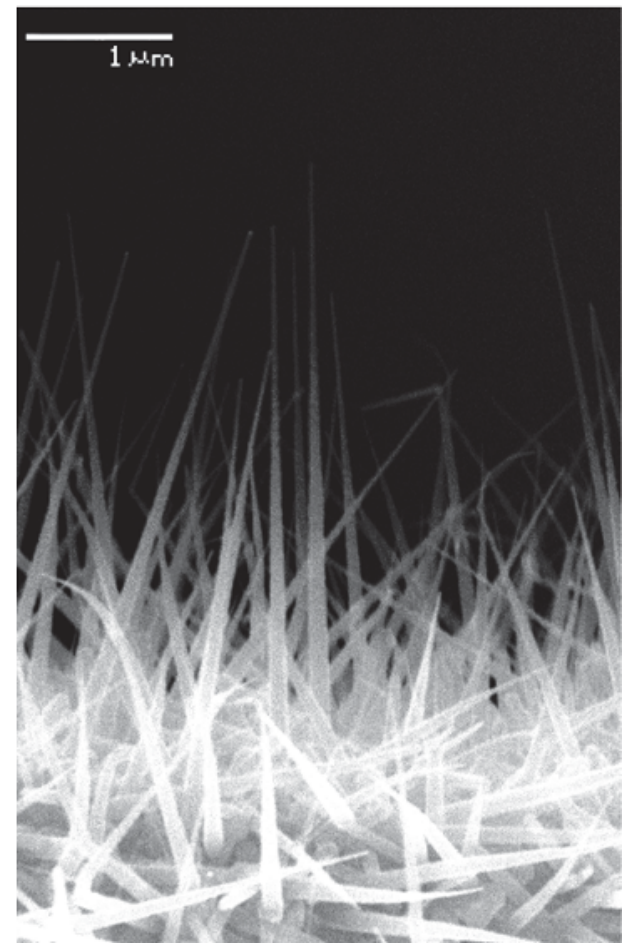

Fig. 4. Side view scanning electron micrograph images of GeNWs on Kapton.

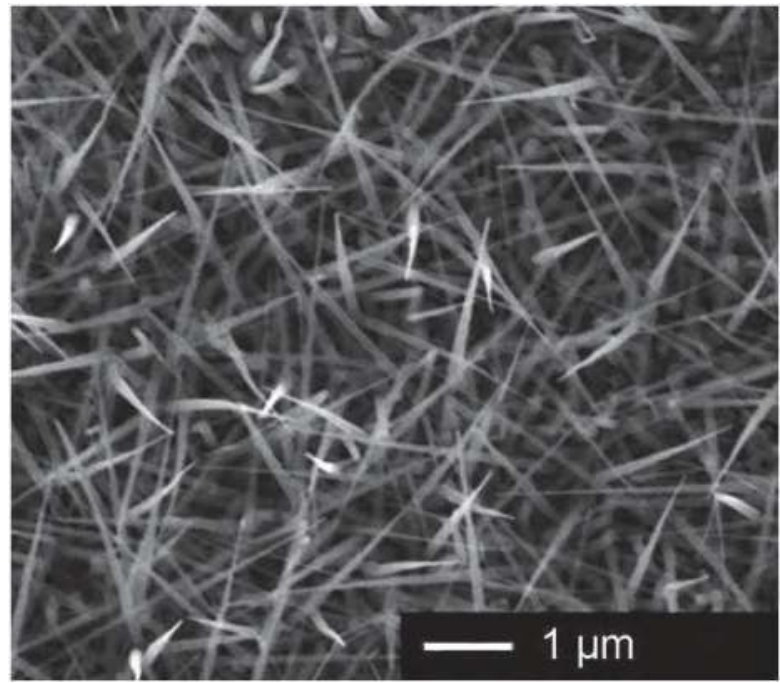

Fig. 5. Top view scanning electron micrograph image of GeNWs grown on poly[ $\left(4,4^{\prime}-\right.$ hexafluoroisopropylidene) diphthalic anhydride-alt-3,6-diaminoacridine hemisulfate]. 


\subsection{Colloidal gold vs. sputtered gold films}

The starting gold catalyst is critical to the nanowire formation. As described in the methods section above, both sputter-coated Au films and wet chemical deposition of colloidal gold particles were utilized as sources of gold. For most applications in nanotechnology it is uniformity in size and spatial distribution which is sought in these wires; variations in length and diameter lead to variations in electrical physical properties and physical. Thus, the ideal growth will produce wires as similar in length and diameter as possible.

To ensure uniformity of nanowire diameter across a single sample during growth, colloidal catalysts are preferred over sputter-coated films. As the eventual nanowire diameter is determined by the prepared colloid diameter, wires can be grown over large areas with precise control over their size. We used predominantly $10 \mathrm{~nm}$ and $20 \mathrm{~nm}$ colloids in these studies. The colloids, as purchased, have a rough concentration of about $10^{11}$ nanoparticles/mL and will generally coat the substrates in a uniform manner when prepared as described above. Less dense areas can be prepared by diluting colloid solutions with distilled, deionized water.

In Figure 6, shown below, the wires were grown from $20 \mathrm{~nm}$ colloids under standard growth conditions as described above. One will note the general consistency among wire diameters. Prior to heating, the colloids are certified by Ted Pella to be within 18 and $22 \mathrm{~nm}$ in diameter. It is observed, however, that wires often differ from this initial diameter range by 10-20 nanometers. There are two reasons for this discrepancy. First, once exposed to air, wires typically grow a $5 \mathrm{~nm}$ coating of native oxide, $\mathrm{GeO}_{x}$, making them appear fatter in scanning electron micrographs.

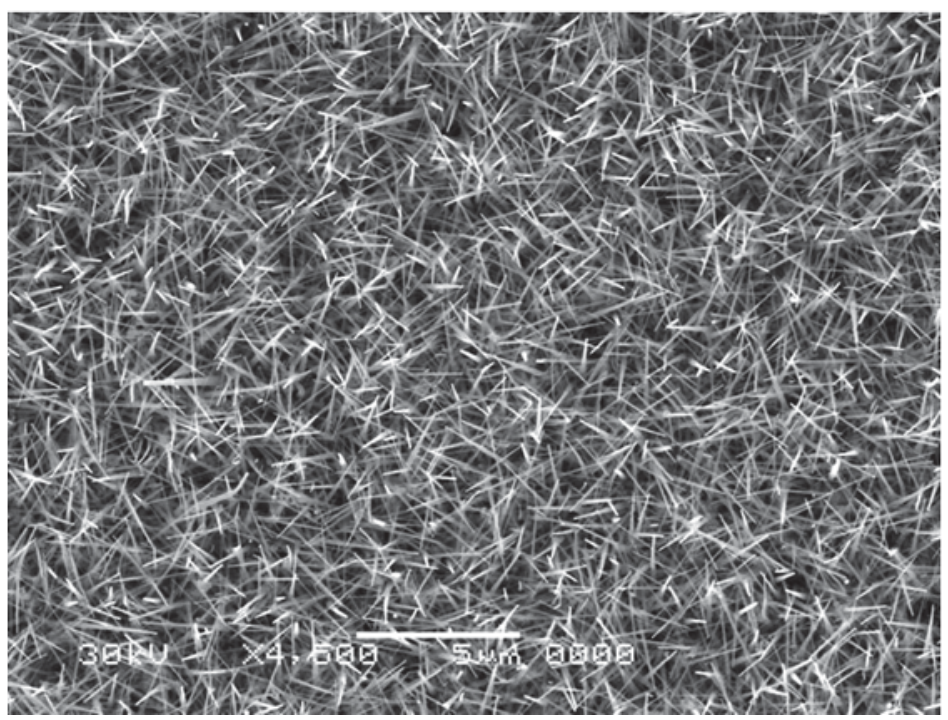

Fig. 6. Germanium nanowires grown from colloidal gold particles attached to the surface through the previously described wet chemical method. Wires are largely consistent in size and length.

Second, once heated, the gold becomes mobile on the substrate surface and can aggregate. Organic linker molecules used to hold the nanoparticles in place vaporize as soon as the 
temperature reaches $213^{\circ} \mathrm{C}$. Heating of the substrate must continue, however, until the bulk gold-germanium eutectic temperature is reached. During this period of time, the wires grown from $20 \mathrm{~nm}$ colloid particles may end up being closer to $40 \mathrm{~nm}$ in diameter, due to the combination of several original particles. There are, however, no such rules or generalizations which can be made for sputter-coated films.

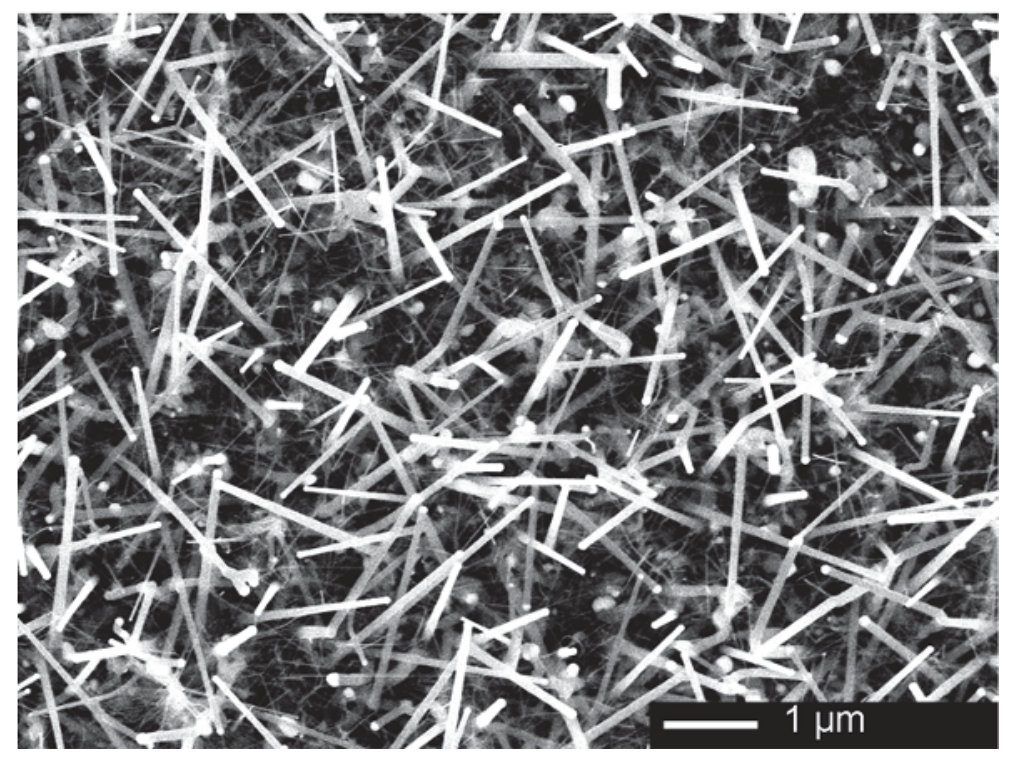

Fig. 7. Scanning electron micrograph of germanium nanowires grown on Kapton from 6.7 $\mathrm{nm}$ gold film. Note the non-uniform distribution of diameters present. Wires were grown under standard conditions.

Figure 7 is a characteristic image consistent with nanowire growth from a sputter-deposited gold film. The wires differ drastically in diameter, with fatter wires reaching several hundred nanometers in diameter and thinner wires maintaining diameters of $50 \mathrm{~nm}$ or smaller. As can be seen from a comparison of Figures 6 and 7, the nanowires grown from colloids are significantly more uniform than those that were grown from the sputter-coated films.

The growth on sputter-deposited films results from the diffusion and nucleation of the gold on the substrate surface. A discrete island or particle of gold is required to begin nucleation of the germanium nanowire. It is well known that when heated, thin gold films become mobile resulting in the balling up of the metal on the surface. It is this mechanism which makes it possible to grow wires from an initially contiguous film over the surface of a substrate. There are, however, specific limitations as to the deposited thickness of this film. Of the sputter-coated films, reasonable nanowire growth was observed for approximately 5$20 \mathrm{~nm}$ thick gold films, area averaged.

If insufficient gold is present, very poor growth is observed, resulting in few, if any, actual wires, as can be seen in Figure 8. For this exceptionally thin coating, it appears there is not enough gold to form the necessary nucleating islands. Wire-like masses are observed, but 
they exist in the plane of the surface and are not free standing, resembling more amorphous germanium masses capped with gold than any useful nanowire structure.

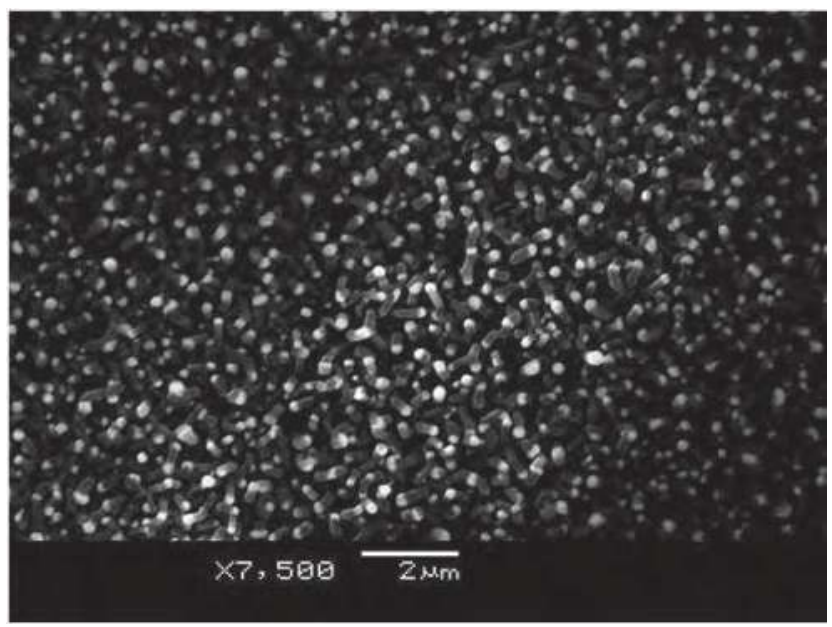

Fig. 8. Poor growth results from $2.8 \mathrm{~nm}$ gold film. "Wires" were grown under standard conditions.

If too much gold is present, an equally significant problem is encountered. Eventually, with the increase of thickness, one approaches a film which acts more closely to a bulk layer of gold. Balling-up and mobility across the surface is hindered, and catalyst islands do not form, making it impossible for nanowire nucleation to begin. As can be seen in Figure 9, no growth is observed under these conditions. If sputter coating is the only available method of functionalizing the substrate surface with the gold catalysts, it is preferable that the thickness of the deposited gold film is tightly controlled to approximately $10 \mathrm{~nm}$.

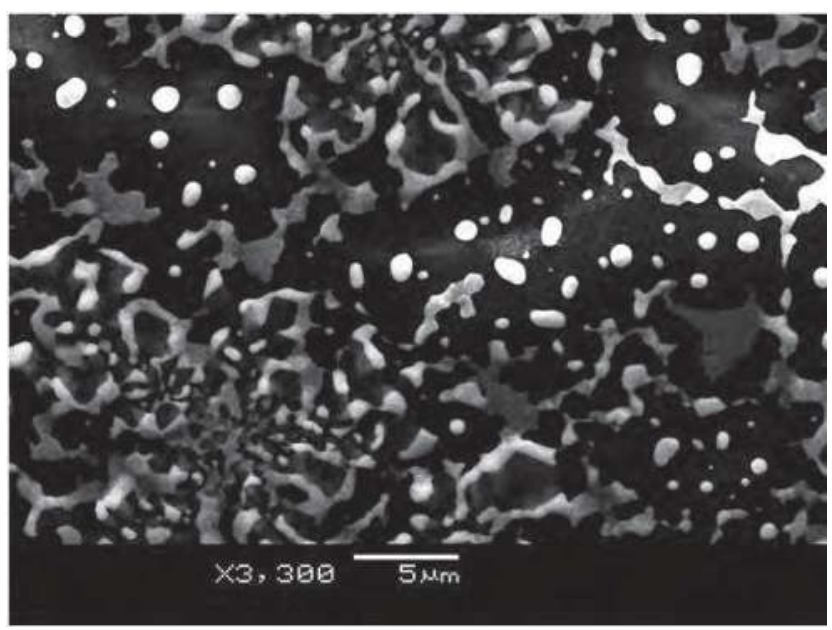

Fig. 9. Poor growth results from a $77 \mathrm{~nm}$ gold film. 
The thicknesses of these films were confirmed through analysis by Rutherford Backscattering Spectroscopy (RBS) as shown in Figure 10.

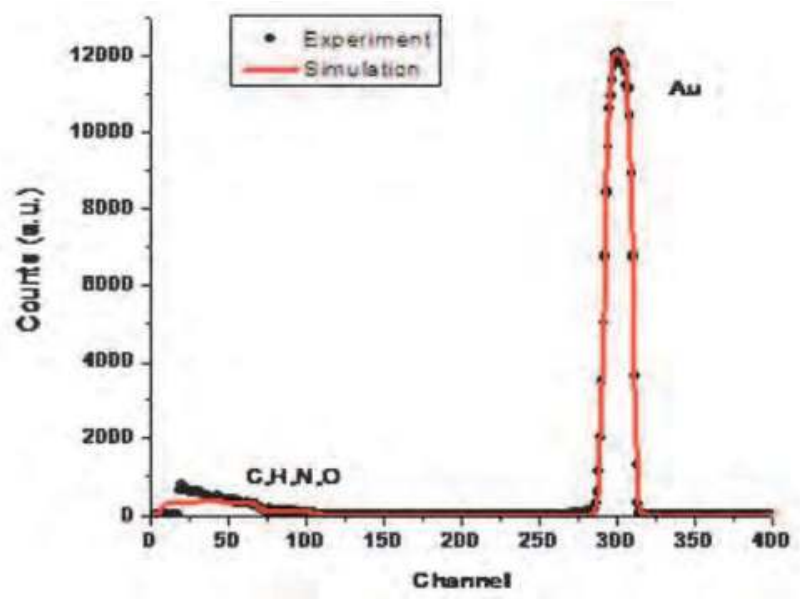

Fig. 10. (a)

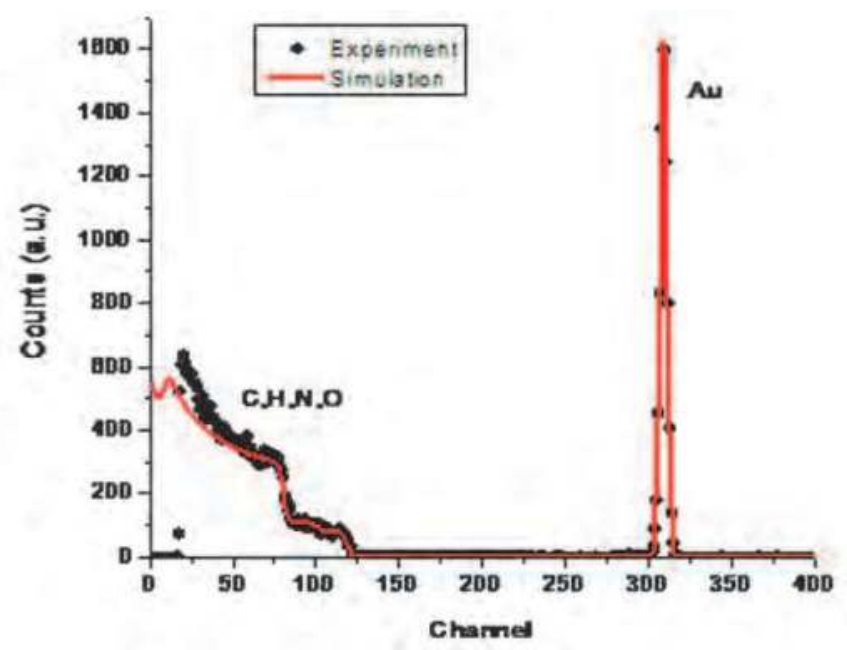

Fig. 10. (b)

Figure 10- RBS spectra confirming an area averaged Au thickness on Kapton. A) $77 \mathrm{~nm} \mathrm{~B}$ ) $2.8 \mathrm{~nm}$

\subsection{AFM studies of Kapton}

At a germanium nanowire growth temperature of $360^{\circ} \mathrm{C}$ (as used in part of this work), the growth temperature approaches the glass transition temperature of Kapton. It was necessary to determine if this would have any effect on the gold catalyst particles deposited 
on the surface during the pre-treatment, particularly if the gold could become embedded within the polymer during the initial heating. In order to examine the location and morphology of the gold nanoparticle catalysts under growth conditions, atomic force microscopy (AFM) was employed. AFM images were obtained using a Digital Instruments MultiMode AFM with a Nanoscope IV controller operating in TappingMode. VISTAprobes non-contact mode Si cantilevers with a spring constant of $0.40 \mathrm{~N} / \mathrm{m}$, resonant frequency of $300 \mathrm{kHz}$, and nominal tip radii of $10 \mathrm{~nm}$ were used.

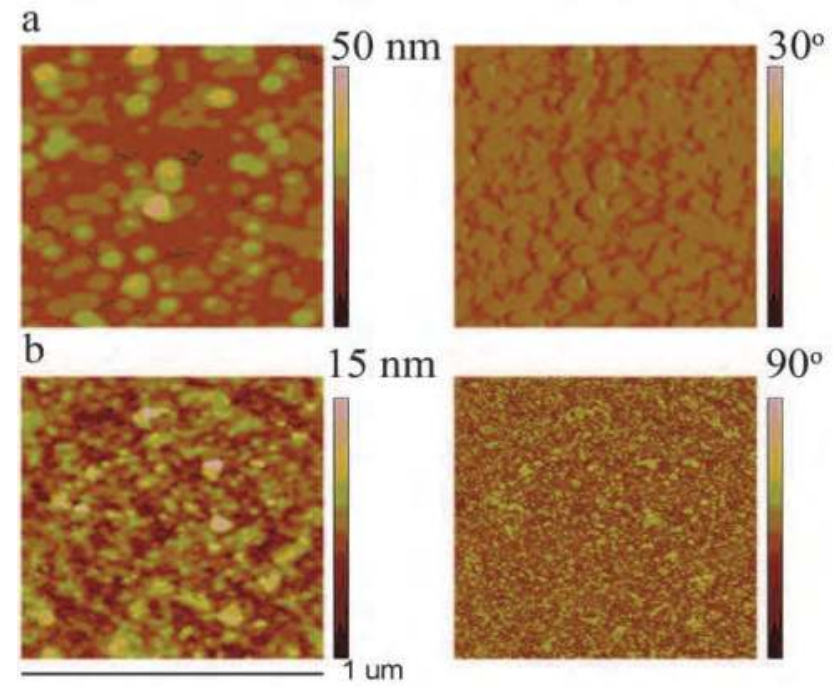

Fig. 11. AFM height (left) and phase (right) images of colloidal Au particles on a Kapton film (a) and plain Kapton film (b) after heating samples under conditions simulating nanowire growth. All images are $1 \mu \mathrm{m}^{2}$.

Scans were performed on Kapton films treated with 3-aminopropyltriethoxysilane (APTES) and colloidal gold particles of 50nm in diameter, as described in the methods section, and an untreated Kapton film as a control. Both samples were imaged before and after heating in $\mathrm{N}_{2}\left(360{ }^{\circ} \mathrm{C}, 15 \mathrm{~min}\right.$.) to mimic the conditions present during nanowire growth. Figure 11 reveals that gold remains in colloidal form on the surface of the polymer and does not appear to migrate into the bulk of the polymer.

Upon heating, the colloids do appear to aggregate and form particles roughly twice their initial diameter. As can be seen from Figure 11a, the colloids appear to be roughly $100 \mathrm{~nm}$ in diameter post-heating. Aggregation of the colloids is expected due to the increasing mobility of gold on surfaces at higher temperatures. This, in turn, is consistent with the typical observation of nanowires possessing diameters larger than the initial catalyst size.

\subsection{Treatment to remove nanowire oxide}

Due to the poor electrical properties of the native oxide of germanium it is a significant problem which must be addressed with in order to utilize germanium nanowires to their full potential in electrical devices. The oxide must be prevented from growing or removed 
after it has developed. Under ambient conditions, germanium surfaces undergo rapid oxidation to $\mathrm{GeO}$ followed by conversion to the thermodynamically more stable $\mathrm{GeO}_{2}$. This oxide can be removed through traditional processing methods such as treatment with a dilute aqueous HF solution. (Adhikari 2005) Our studies show that the germanium oxides are readily removed in a $2 \% \mathrm{HF}$ solution for 1 minute with minimal damage to the GeNWs or the polymer substrate.

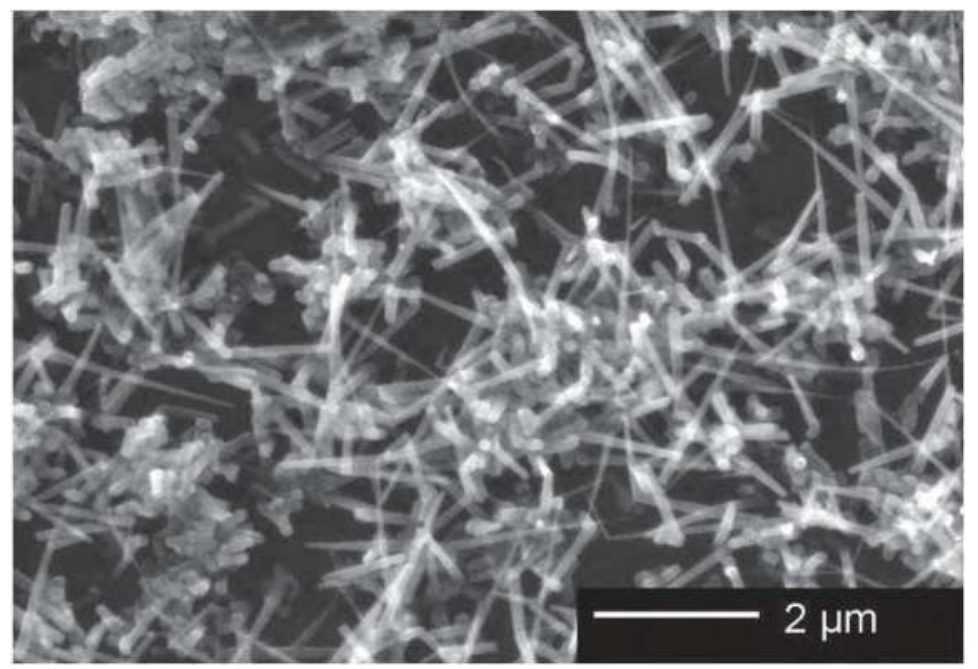

Fig. 12. Nanowires grown on a Kapton substrate retain their integrity after an HF dip.

The chemical robustness of the polymer substrate is key element if the eventual goal for such a system is printable electronics. Flexible polymer sheets and any deposited components must be able to withstand wet chemical processing to be of use. As can be seen in Figure 12, nanowires exposed to the HF treatment described above retain their structure and attachment to the Kapton surface.

\subsection{Passivation}

One method to passivate germanium involves capping the surface with organic thiols. Germanium nanowire samples grown on polymers were placed in a $0.1 \mathrm{M}$ solution of 1octanethiol for 24 hours immediately upon removal from the reactor. To confirm the level of oxide on the surface, X-ray photoelectron spectroscopy was used. For the following spectra, data were collected using $\mathrm{Al} \mathrm{Ka}$ anode source with a cylindrical mirror analyzer. The FWHM of the C 1s peak (2.4 eV) was used as the FWHM for the deconvolution of the Ge $2 p$ peaks. The $C 1$ s is a single Gaussian and represents the energy resolution of the instrument at the pass energy utilized. Within the deconvolution of the Ge $2 p 1 / 2$, the peak with the lowest binding energy was assigned to that of bulk Ge. All spectra were shifted to ensure that this peak was centered at $1217.0 \mathrm{eV}$.

A comparison of passivated and non-passivated samples reveals a substantial decrease in the surface oxidation for samples that had been thiol passivated. Non-passivated samples possess a substantial oxide layer consisting primarily of $\mathrm{GeO}_{2}$, with a smaller contribution from $\mathrm{GeO}$, as can be seen in Figure 13a. The passivated species also exhibit a small higher 
binding energy shoulder consisting of a single peak attributed primarily to $\mathrm{GeO}$, as can be seen in figure $13 \mathrm{~b}$. However, the contribution relative to the bulk germanium peak is greatly reduced from that of the non-passivated sample. The presence of $\mathrm{GeO}$ suggests that even the brief exposure of the sample to atmosphere while transferring from the growth chamber to the thiol solution is enough to partially oxidize the germanium. However, as previously mentioned, the stability of the polymer substrate is sufficient to remove this oxide coating prior to passivation, thus greatly reducing the presence of oxide.
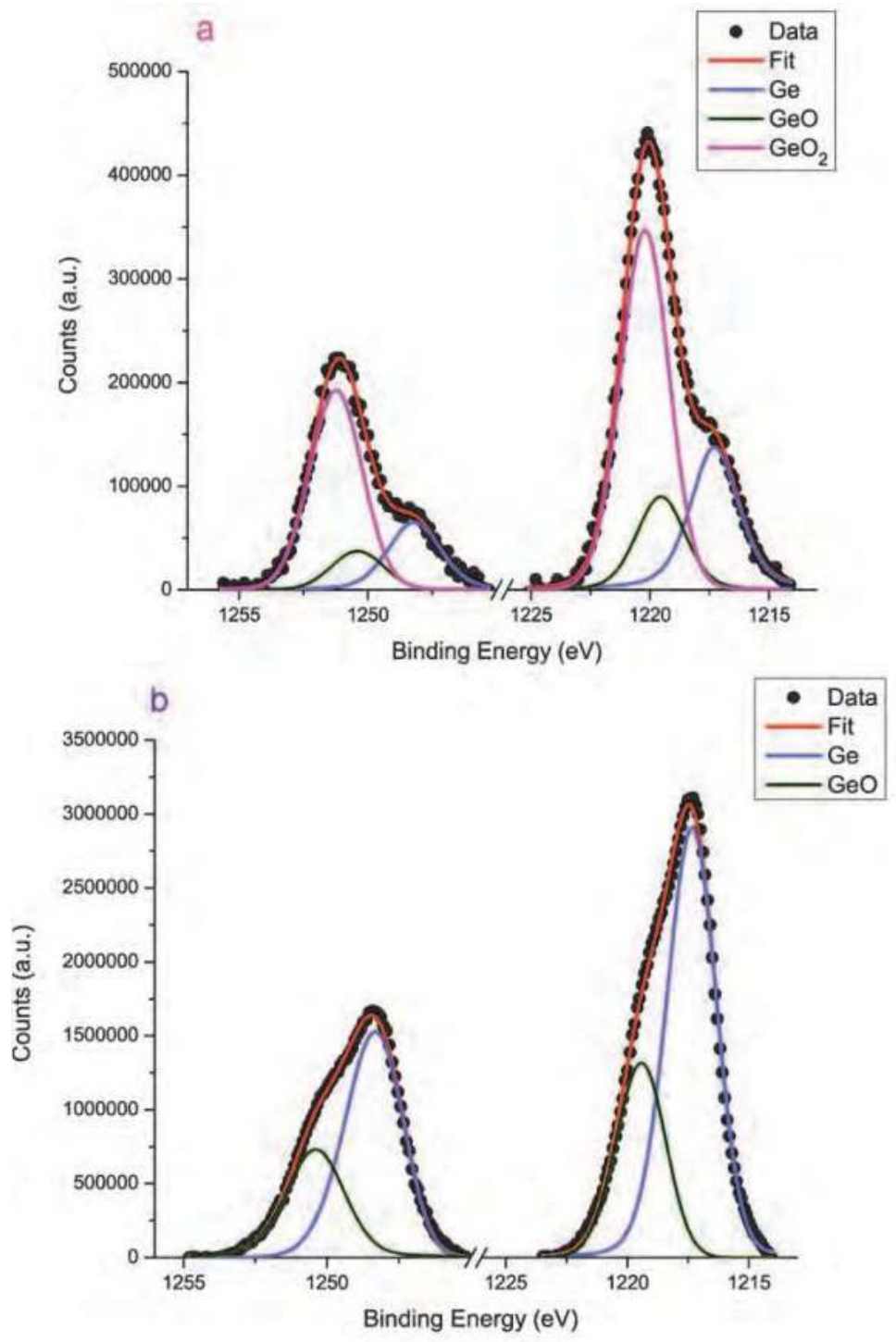

Fig. 13. XPS spectra of Ge 2p3/2 and 2p1/2 for non-passivated (a) and passivated (b) GeNWs 
Passivation between processing steps, and passivation/packaging at the end of processing, are key in realizing device applications. The growth, processing, and passivation methods detailed in this work are all found to lead to a flexible and well-integrated GeNW-polymer hybrid film, of potential importance for a range of flexible applications such as batteries, solar cells, sensors, and transistors.

\section{Conclusion}

In conclusion, we present results of GeNW VLS growth on flexible organic substrates. The nanowire attachment to the polymer substrates is robust and able to withstand flexing of the substrate. Post-growth processing to remove the oxide and to functionalize the nanowires (with or without the oxide present), can be applied without damaging the substrate. This work is of potential importance for a range of flexible applications such as batteries, solar cells, sensors and transistors. Direct growth of nanowires on flexible substrates simplifies processing and improves yield. We compare various methods of catalyst preparation from which nanowire growth can be initiated, and conclude that wet chemical methods to deposit colloidal gold particles on the substrate surface produces wires superior in uniformity than sputter coated gold films.

\section{Acknowledgements}

The authors thank the National Science Foundation for financial support for this work. They acknowledge Ozgur Celik, Leszek Wielunski, and Robert Lorber for their valuable time and advice.

\section{References}

Adhikari, H., A. F. Marshall, et al. (2006). Nano Lett. 6(2): 318-323.

Adhikari, H., A. F. Marshall, et al. (2007). ACS Nano 1(5): 415-422.

Adhikari, H., Sun, S., McIntyre, P., and Chidsey, C.E.D (2005). Applied Physics Letters 87.

Allen, P. B. (2007). Nano Lett. 7(1): 6-10.

Bitetti, G., M. Marchetti, et al. (2007). Acta Astronautica 60(3): 166-174.

Bradley, K., J.-C. P. Gabriel, et al. (2003). Nano Lett. 3(10): 1353-1355.

Choi, H., I. S. Chung, et al. (2008). Polymer.

Chung, C.-L. and S.-H. Hsiao (2008). Polymer 49(10): 2476-2485.

Dai, H. (2005). Angew. Chem. Int. Ed. 44: 2-5.

Dailey, J. W., Taraci, J., Clement, T., Smith, D.J., and Picraux, S.T. (2004). J. Appl. Phys 96.

Fan, Z., J. C. Ho, et al. (2008). Nano Lett. 8(1): 20-25.

Gibson, J., and Daghlian, C.P. (2005). J. Appl. Phys 97.

Greytak, A. B., Lauhoun, L. J., Gudiksen, M.S., and Lieber, C.M. (2004). Applied Physics Letters 84 .

Hanrath, T. and B. A. Korgel (2002). J. Am. Chem. Soc. 124(7): 1424-1429.

Hanrath, T. and B. A. Korgel (2004). J. Am. Chem. Soc. 126(47): 15466-15472.

Heath, J. (2000). J. Phys. Chem 104(11864-11870).

Jing, M., M. Ni, et al. (2006). J. Phys. Chem. B 110(37): 18332-18337.

Kang, K., Lee, H., Kim, C., Yang, J., and Jo, M. (2008). Advanced Materials 9999. 
Li, H., J. Liu, et al. (2006). Polymer 47(4): 1443-1450.

Lin, C., Liao, H., and Chen, S (2005). Applied Physics Letters(86).

Maya, E. M., A. E. Lozano, et al. (2005). Polymer 46(25): 11247-11254.

McAlpine, M. C., R. S. Friedman, et al. (2003). Nano Lett. 3(11): 1531-1535.

Pasquier, A. D., D. D. T. Mastrogiovanni, et al. (2007). Applied Physics Letters 91(18): 183501.

Ree, M., K. Kim, et al. (1997). Journal of Applied Physics 81(2): 698-708.

Sang, W., Han, M., Carraro, C., and Maboudian, R., (2001). J. Am. Chem. Soc. 123.

Saran, N., K. Parikh, et al. (2004). J. Am. Chem. Soc. 126(14): 4462-4463.

Wang, D., Y.-L. Chang, et al. (2005). J. Am. Chem. Soc. 127(33): 11871-11875.

Wang, D., Y.-L. Chang, et al. (2004). J. Am. Chem. Soc. 126(37): 11602-11611.

Wang, L., B. L. Yi, et al. (2008). J. Phys. Chem. B 112(14): 4270-4275.

Wu, Y. and P. Yang (2000). Chem. Mater. 12(3): 605-607.

Yun, M. (2003). Nanofabrication Technologies 5220. 


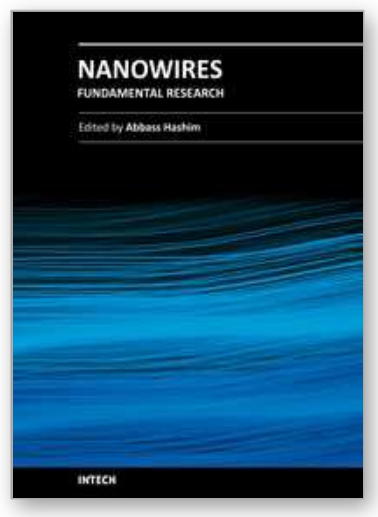

\author{
Nanowires - Fundamental Research \\ Edited by Dr. Abbass Hashim
}

ISBN 978-953-307-327-9

Hard cover, 552 pages

Publisher InTech

Published online 19, July, 2011

Published in print edition July, 2011

Understanding and building up the foundation of nanowire concept is a high requirement and a bridge to new technologies. Any attempt in such direction is considered as one step forward in the challenge of advanced nanotechnology. In the last few years, InTech scientific publisher has been taking the initiative of helping worldwide scientists to share and improve the methods and the nanowire technology. This book is one of InTechâ $€^{\mathrm{TM}_{\mathrm{S}}}$ attempts to contribute to the promotion of this technology.

\title{
How to reference
}

In order to correctly reference this scholarly work, feel free to copy and paste the following:

Lauren Klein, Daniel Mastrogiovanni, Alan Wan and Eric Garfunkel (2011). Growth of Germanium Nanowires on a Flexible Organic Substrate, Nanowires - Fundamental Research, Dr. Abbass Hashim (Ed.), ISBN: 978953-307-327-9, InTech, Available from: http://www.intechopen.com/books/nanowires-fundamentalresearch/growth-of-germanium-nanowires-on-a-flexible-organic-substrate

\section{INTECH}

open science | open minds

\section{InTech Europe}

University Campus STeP Ri

Slavka Krautzeka 83/A

51000 Rijeka, Croatia

Phone: +385 (51) 770447

Fax: +385 (51) 686166

www.intechopen.com

\section{InTech China}

Unit 405, Office Block, Hotel Equatorial Shanghai

No.65, Yan An Road (West), Shanghai, 200040, China 中国上海市延安西路65号上海国际贵都大饭店办公楼 405 单元 Phone: $+86-21-62489820$

Fax: $+86-21-62489821$ 
(C) 2011 The Author(s). Licensee IntechOpen. This chapter is distributed under the terms of the Creative Commons Attribution-NonCommercialShareAlike-3.0 License, which permits use, distribution and reproduction for non-commercial purposes, provided the original is properly cited and derivative works building on this content are distributed under the same license. 